\title{
Is the psoas a hip flexor in the active straight leg raise?
}

\author{
Hai Hu • Onno G. Meijer · Jaap H. van Dieën · Paul W. Hodges $\cdot$ \\ Sjoerd M. Bruijn • Rob L. Strijers · Prabath W. B. Nanayakkara • \\ Barend J. van Royen · Wen Hua Wu Chun Xia
}

Received: 23 December 2009/Revised: 17 May 2010/ Accepted: 27 June 2010/Published online: 13 July 2010

(C) The Author(s) 2010. This article is published with open access at Springerlink.com

\begin{abstract}
Psoas function is a topic of considerable relevance in sports and clinical science. However, the literature on psoas function is not sufficiently consistent. Questions are, amongst others, if during hip flexion the psoas always has the same function as the iliacus, and if the psoas affects the hip more than the lumbar spine. In the present study, 17 healthy women, 20-40 years, performed the active straight leg raise (ASLR), with the right or the left leg ("Side"), and without or with weight added above the ankle ("Condition"). Electromyographic (EMG) activity of psoas and iliacus were recorded with fine-wire electrodes, and of rectus femoris and adductor longus with surface electrodes, all on the right side. Movements of the leg were recorded with active markers and a camera system. During ASLR, the iliacus, rectus femoris, adductor longus and psoas were active ipsilaterally, but psoas was also active contralaterally. All muscles started to contract before movement onset, the iliacus, rectus femoris, and adductor longus largely at the same time, before the psoas. There was no
\end{abstract}

H. Hu · O. G. Meijer ( $\square)$ · J. H. van Dieën · S. M. Bruijn Faculty of Human Movement Sciences, Research Institute MOVE, VU University, Van Der Boechorststraat 9,

1081 BT Amsterdam, The Netherlands

e-mail: o_g_meijer@fbw.vu.nl

H. Hu - C. Xia

Department of Orthopaedics, Zhongshan Hospital, Xiamen

University, Xiamen, Fujian, People's Republic of China

O. G. Meijer - W. H. Wu

Second Affiliated Hospital of Fujian Medical University, Quanzhou, Fujian, People's Republic of China

O. G. Meijer - W. H. Wu

Orthopaedic Biomechanics Laboratory of Fujian Medical

University, Quanzhou, Fujian, People's Republic of China significant difference between the amplitude or time of onset of ipsilateral and contralateral psoas EMG activity, nor was there a significant interaction between Side and Condition for the psoas. Although ipsilateral psoas activity is consistent with the psoas being a hip flexor, contralateral activity is not. The most simplest explanation of the pattern found is that the psoas is bilaterally recruited to stabilize the lumbar spine, probably in the frontal plane.

Keywords M. psoas - Hip flexion - Fine-wire EMG . Lumbar spine stability - Active straight leg raise

\section{Introduction}

According to the 40th edition of Gray's Anatomy, "Psoas major ..., together with iliacus, flexes the thigh ..." [22, p. 1368]. The text continues that psoas may be a lateral rotator of the hip, that bilateral psoas action bends the trunk

P. W. Hodges

Centre of Clinical Research Excellence in Spinal Pain, Injury and Health, School of Rehabilitation Sciences,

The University of Queensland, Brisbane, QLD, Australia

R. L. Strijers

Department of Clinical Neurophysiology, VU University

Medical Centre, Amsterdam, The Netherlands

P. W. B. Nanayakkara

Department of Internal Medicine, VU University Medical

Centre, Amsterdam, The Netherlands

B. J. van Royen

Department of Orthopaedic Surgery, VU University Medical

Centre, Amsterdam, The Netherlands 
and pelvis forwards, and that there is no evidence that unilateral psoas action causes lateral or forward flexion of the trunk, although in "symmetrical upright stance, iliopsoas has some action from below to maintain the vertebral column upright." (ibid). In sum, it is suggested that psoas mainly works with iliacus as a hip flexor, with also "some", that is to say: "less", effect on the spine. Theoretically and experimentally, however, these views are not without problems.

Psoas is a long muscle, with fascicles arising at T12-L5 from the bodies of two adjoining vertebrae and their intervertebral discs, as well as fascicles from the L1-L5 transverse processes [6]. Psoas passes the pelvis, and inserts onto the trochanter minor. Owing to its position, it is difficult to investigate psoas non-invasively, and one early study of its function was performed in the framework of lumbar sympathectomy [14]. Since the 1990s, fine-wire electrodes have been inserted via a needle from the dorsal trunk under ultrasound guidance [e.g. 1], and the muscle became more accessible. Still, the literature on psoas function is rife with controversy and contradiction.

About half a century ago, Basmajian argued that psoas and iliacus could not be expected to have a different function, and inferred psoas activity from iliacus electromyography (EMG) [4]. Since then, it has been shown that the two muscles "have individual and task specific activation patterns" [1, p. 10]. Still, even today, authors infer psoas function from surface EMG in the groin [e.g. 8]. Another point of contradiction is Bogduk's view, a main source for Gray's Anatomy [7], that psoas cannot have much effect on the spine, because moment arms are small, and all fascicles have the same length. Bogduk [6] concluded that psoas is "designed to act on the hip". However, this was disputed by Santaguida and McGill [21, p. 345], who argued that the anatomy of the psoas is "ideally suited" for lateral stabilization of the lumbar spine. Finally, most authors agree that psoas activity increases with larger hip flexion [1], while Yoshio et al. [25] even concluded that psoas mainly works as a stabilizer of the lumbar spine and the femoral head in the first $15^{\circ}$ of hip flexion, and does not become an effective hip flexor before $45^{\circ}$ of flexion.

The above debates are far from trivial. Psoas function has drawn much attention in sports [10] and clinical literature $[13,15]$. The present study focuses on psoas function during the active straight leg raise (ASLR). The ASLR involves hip flexion, but also challenges the stability of the lumbar spine due to the large moment of gravity and the effects of muscles [12]. Investigation of the ASLR is also clinically relevant, as ASLR is often limited in pregnancyrelated pelvic girdle pain [26].

\section{Methods}

Participants were healthy $20-40$ years old women with normal blood pressure. In total, 20 participants were recruited. Three participants became light-headed or fainted once standing up 5-10 min after insertion of the psoas electrode, leaving 17 participants. The age, weight, height and BMI of the participants were $28.7 \pm 2.8$ years (mean, SD), $\quad 60.7 \pm 9.7 \mathrm{~kg}, \quad 167.6 \pm 7.5 \mathrm{~cm}$, and $21.5 \pm 2.4$, respectively. Participants gave written informed consent. The protocol was approved by the Local Medical Ethical Committee.

\section{Electromyography}

Muscles were measured on the right side only. For fine-wire EMG, we used CE-marked paired hook-wire electrodes (40 gauge insulated stainless steel, VIASYS Healthcare, Madison WI, USA), threaded into sterile 50 or $100 \mathrm{~mm}$ hypodermic needles, with 5-7 mm long "hooks" extending from the tip. After disinfection, the needles were inserted with ultrasound guidance, under semi-sterile conditions. Iliacus insertion was $1 \mathrm{~cm}$ inferior to the inguinal ligament, $2 \mathrm{~cm}$ medial to a vertical line down from the anterior superior iliac spine, and for the psoas major, 5-8 cm lateral to L3-L4 [1]. The use of ultrasound allowed visual monitoring of the final placement of the fine-wire electrodes. These procedures are regarded as safe, but most of our subjects were anxious about psoas insertion and in about $25 \%$, the needle hit a transverse process, causing some pain [12]. Any symptoms were transient and recovered after the removal of the electrodes; no serious adverse effects were observed.

For surface EMG, pairs of electrodes (10 diameter $\mathrm{Ag}$ / $\mathrm{AgCl}$ discs, inter-electrode distance $20 \mathrm{~mm}$; Kendall ARBO, Neustadt am Dom, Germany) were placed over the rectus femoris and adductor longus [12].

EMG data were amplified 20 times, band-pass filtered between $20 \mathrm{~Hz}$ and $1 \mathrm{kHz}$, and sampled at $2 \mathrm{kHz}$ using a multichannel system (Porti5, TMS-International, Enschede, The Netherlands), with input impedance adapted to the fine wire.

\section{Kinematics}

Four cluster markers were attached to the upper and lower legs. Each cluster marker contained three infrared emitting diodes for movement registration with a $2 \times 3$ camera system (OPTOTRAK 3020, Northern Digital, Waterloo, ON, Canada), connected via a synchronisation cable to the Porti. For kinematics, the sampling frequency was $50 \mathrm{~Hz}$. 


\section{Conditions}

In right and left ASLR, subjects were supine, their legs straight and feet in dorsiflexion, $20 \mathrm{~cm}$ apart [16]. They were instructed to raise each leg three times until the foot reached $20 \mathrm{~cm}$ above the table, without bending the knees, and keeping the leg elevated for $10 \mathrm{~s}$.

The whole procedure was repeated with weight added just above the ankle. Using lower extremity anthropometry and specific regression equations [27, p. 605], the mass of this weight was calculated in such a way that the static moment of the leg with respect to the hip was increased by $50 \%$.

\section{Data analysis}

Data were analysed with MATLAB 7.4 (The Mathworks, Nattick, MA, USA). Kinematic data were filtered with a 4th order bi-directional low-pass Butterworth filter with a cutoff frequency of $5 \mathrm{~Hz}$. Onset and peak of leg raise were derived (first point with zero velocity before/after a peak in velocity), as was leg raise velocity (height of peak position divided by time to reach peak position). From the markers in the relevant clusters, average heights of the upper and lower legs were calculated over the three repetitions per subject per condition.

Fine-wire EMG was not usable from four subjects. Further, two sets of iliacus data contained too much noise, particularly during the condition with weight added; these data were removed from amplitude calculations (leaving, for amplitude, $N=13$ for the psoas, iliacus $N=11$, rectus femoris and adductor longus $N=17$ ). The onset signal was too noisy in three sets of psoas data, and these were removed from onset calculations. Synchronisation failed to work three times, rendering onset determination impossible, which led to the removal of one more set of psoas data, and three sets of rectus femoris and adductor longus data (leaving, for onset, $N=9$ for the psoas, iliacus $N=11$, rectus femoris and adductor longus $N=14)$.

All EMG data were high-pass filtered bi-directionally at $250 \mathrm{~Hz}$ (first-order Butterworth), to remove ECG contamination and to obtain more precise (less variable) estimates of the EMG amplitude [20, 24], then full-wave rectified, and low-pass filtered bi-directionally at $5 \mathrm{~Hz}$ (second-order Butterworth). Median EMG amplitude during plateau of movement (5-10 s after movement onset) was calculated. Median onset time was determined by means of a dedicated algorithm (using the approximated generalised likelihood ratio [23]), where necessary corrected on the basis of visual inspection [11]. EMG onset was expressed in relation to movement onset.
Statistical analysis

Statistical analysis was performed with SPSS 16, with $P<0.05$ as threshold for significance. Generalised estimation equations (GEEs) on the amplitude of muscle activity were used, with Side (ipsilateral vs. contralateral) and Condition (without vs. with weight added) as factors. Then, GEEs were performed on onset time of muscle activity, for all relevant muscle pairs, with Muscle (the one vs. the other in the pair in question) and Condition (without vs. with weight added) as factors. GEEs calculate regression equations on the basis of repeated measures, while allowing for missing values.

\section{Results}

Heights reached by the upper/lower leg were not significantly affected by Side (right/left ASLR) or Condition (without/with weight), nor was there a significant Side $\times$ Condition interaction. Further, the velocity of the leg raise was not significantly affected by Side, Condition, or their interaction.

Amplitude of muscle activity

During right or left ASLR (Fig. 1), there was ipsilateral activity in all four muscles. Psoas was also active during contralateral ASLR.

In GEEs on amplitude (Table 1), an effect of Side was found for iliacus, rectus femoris, and adductor longus $(P=0.00)$, with more activity during ipsilateral ASLR. There was no effect of Side for the psoas $(P=0.98)$. All four muscles were significantly more active with weight $(P=0.00)$. For iliacus, rectus femoris, and adductor longus, there were significant Side $\times$ Condition interactions $(P=0.00)$, with more activity during ipsilateral ASLR with weight added. No such interaction was found for psoas $(P=0.95)$.

\section{Onset of muscle activity}

Given the lack of clear muscle activity in the contralateral iliacus, rectus femoris, and adductor longus, only ipsilateral activity of these muscles was used for onset analysis.

EMG activity typically increased before onset of leg elevation (Figs. 2, 3). GEEs of onset time (Table 2), ipsilaterally, revealed that iliacus, rectus femoris, and adductor longus EMG increased before that of psoas (muscle, $P=0.01)$. Onset of ipsilateral iliacus, rectus femoris, and adductor longus EMG was largely synchronous, with one exception, adductor longus EMG increased before iliacus in the condition with weight $(P=0.02)$. There was no 
Fig. 1 EMG activity $(\mu \mathrm{V})$ of the psoas, iliacus, rectus femoris, and adductor longus, during contralateral and ipsilateral ASLR without (grey) or with (black) weight added. Error bars represent standard deviations

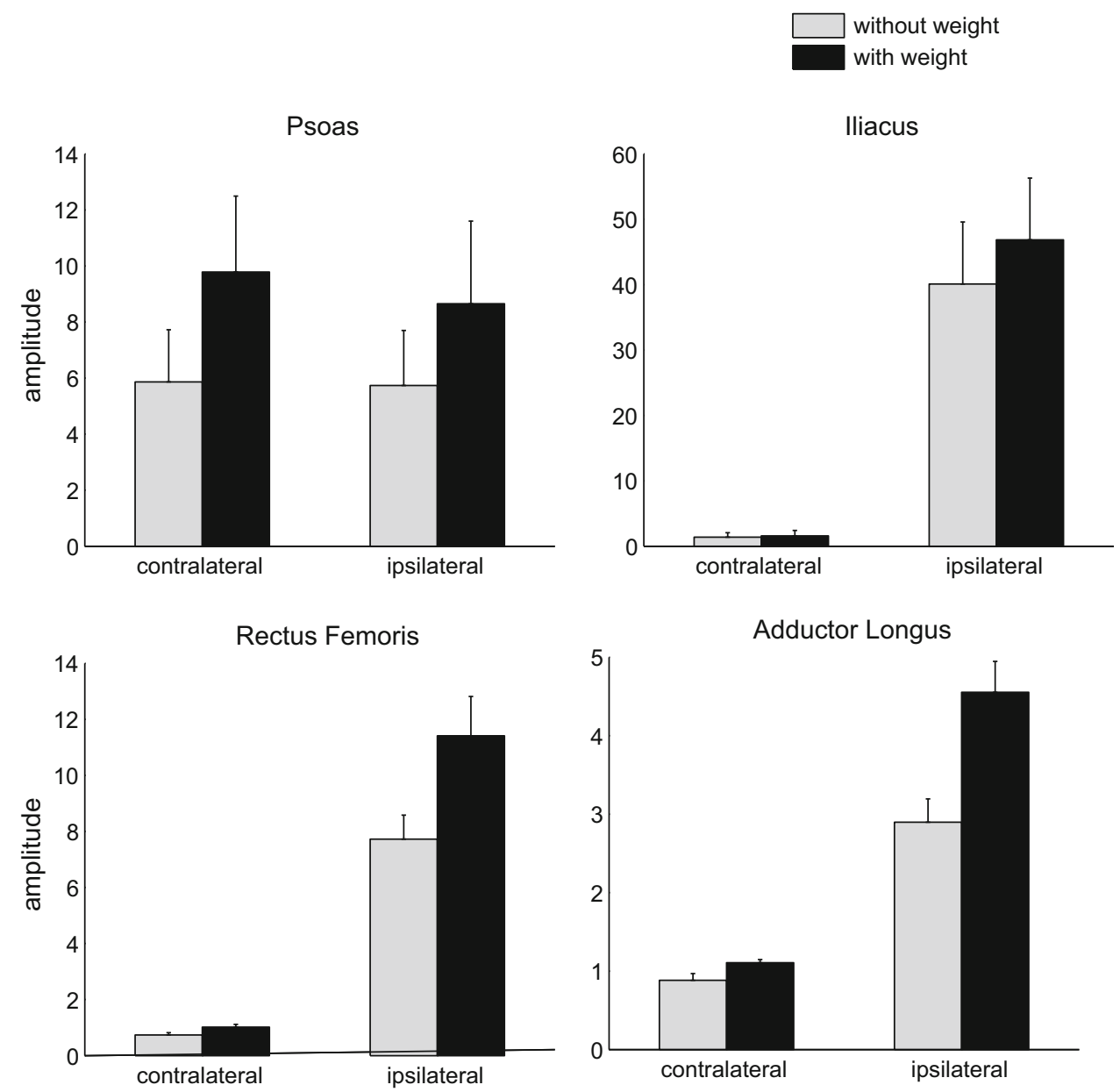

Table 1 Regression coefficients (B) and $P$ values from GEEs on amplitudes of right-sided muscle activity during the active straight leg raise (ASLR), with Side (ipsilateral vs. contralateral) and Condition (without vs. with weight added above the ankle) as factors

\begin{tabular}{|c|c|c|c|c|c|c|c|c|}
\hline \multirow[t]{2}{*}{ Amplitude $(\mu \mathrm{V})$} & \multicolumn{2}{|c|}{ Intercept } & \multicolumn{2}{|l|}{ Side } & \multicolumn{2}{|c|}{ Condition } & \multicolumn{2}{|c|}{ Interaction } \\
\hline & $B$ & $P^{\mathrm{a}}$ & $B^{\mathrm{b}}$ & $P^{\mathrm{a}}$ & $B^{\mathrm{c}}$ & $P^{\mathrm{a}}$ & $B^{\mathrm{d}}$ & $P^{\mathrm{a}}$ \\
\hline Psoas & 6.41 & 0.00 & -0.08 & 0.98 & 3.98 & 0.00 & & \\
\hline Iliacus & 1.44 & 0.00 & 38.66 & 0.00 & 0.15 & 0.00 & 6.61 & 0.00 \\
\hline Rectus femoris & 0.74 & 0.00 & 6.99 & 0.00 & 0.28 & 0.00 & 3.41 & 0.00 \\
\hline Adductor longus & 0.88 & 0.00 & 2.01 & 0.00 & 0.23 & 0.00 & 1.43 & 0.00 \\
\hline
\end{tabular}

Non-significant interactions have been left out

Note that GEE calculates regression equations, and, for instance, the first line reads as: psoas activity equals $6.41+$ (in conditions with weight) 3.98 (and some non-significant components)

${ }^{a}$ For the model effects (which may be different from $P$ values for specific parameterizations)

${ }^{\mathrm{b}}$ For the ipsilateral side (i.e. right side ASLR)

${ }^{c}$ For the condition with weight added

d Ipsilateral, with weight

significant difference between ipsilateral and contralateral psoas onset times $(P=0.15)$. EMG onset of all muscles was earlier when weight was added $(P \leq 0.04)$.

\section{Discussion}

Muscle activity was recorded of psoas and iliacus (finewire EMG electrodes), as well as rectus femoris and adductor longus (surface EMG electrodes), during an ASLR, without or with a weight added above the ankle. All muscles showed task-related activity. Iliacus, rectus femoris, and adductor longus were active during ipsilateral ASLR only, whereas psoas was also contralaterally active. Onset of muscle activity preceded leg elevation, but was somewhat later for psoas than for the other three muscles. Muscles were more active, and started their activity earlier, when weight was added. Psoas EMG amplitude and onset time were, on average, not different between ipsilateral and contralateral ALSR, and there was also no interaction between Side (ipsilateral vs. contralateral) and Condition (without or with weight added).

Hip flexion

As psoas passes anterior to the flexion/extension axis of the hip on its way to the trochanter minor, mechanically, psoas 

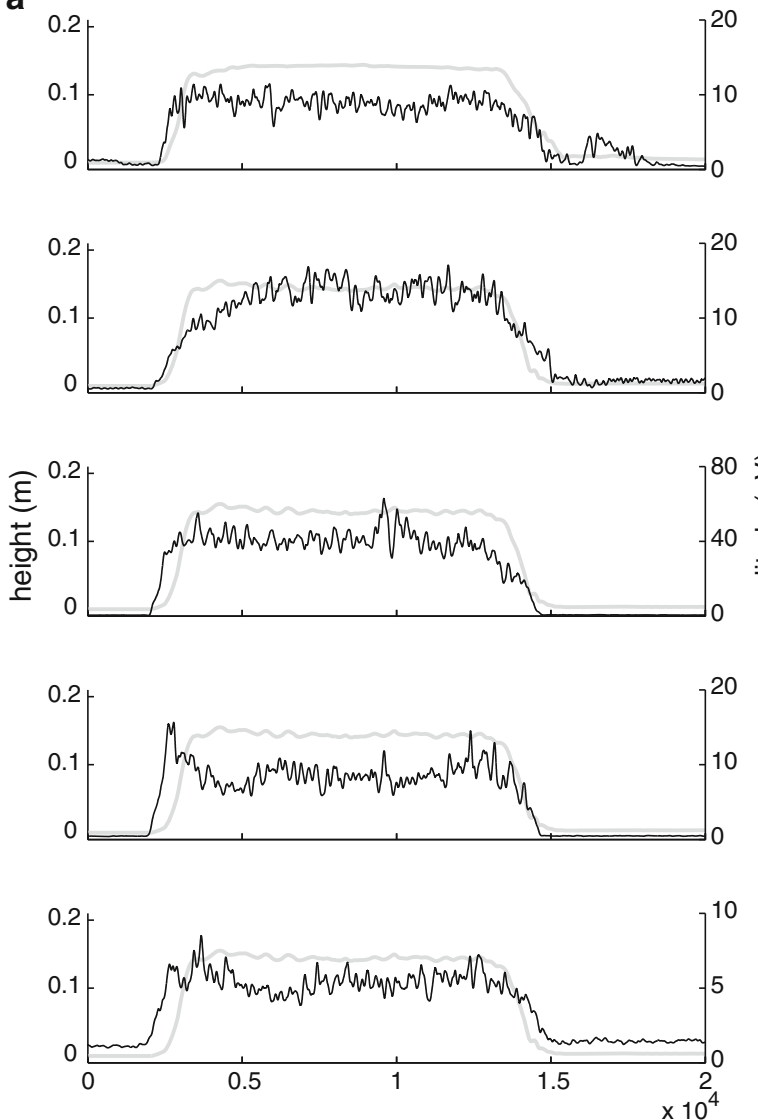

Fig. 2 a Representative example, in a condition with weight added, of muscle activity $(\mu \mathrm{V}$, right vertical axis, drawn in black) during ASLR. Movement of the lower leg is also depicted ( $\mathrm{m}$, left vertical axis, drawn in grey). Time (ms) is given on the horizontal axis. For

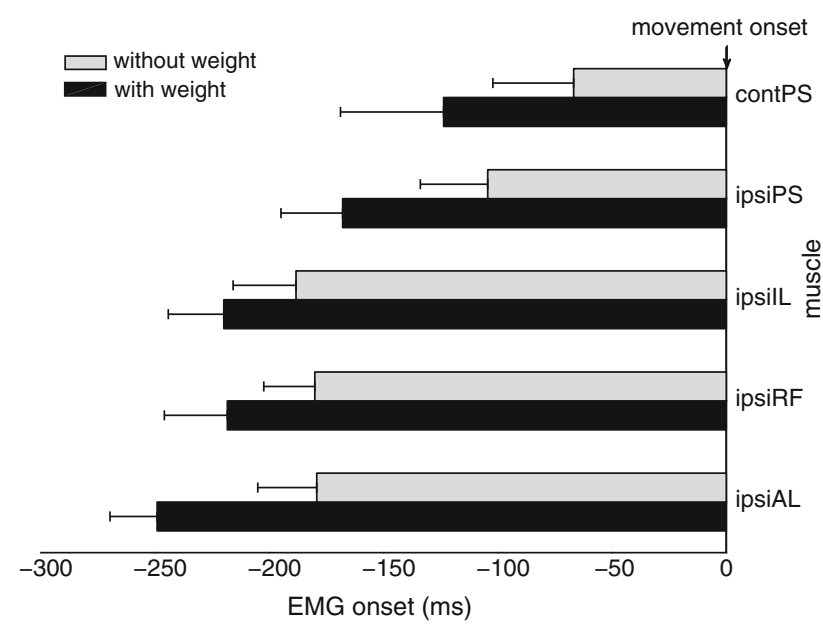

Fig. 3 Time of onset of EMG activity during the active straight leg raise (ASLR), before the onset of elevation of the lower leg, in the contralateral psoas (contPS), the ipsilateral psoas (ipsiPS), the ipsilateral iliacus (ipsiIL), the ipsilateral rectus femoris (ipsiRF), and the ipsilateral adductor longus (ipsiAL), in conditions without (grey) and with (black) weight added
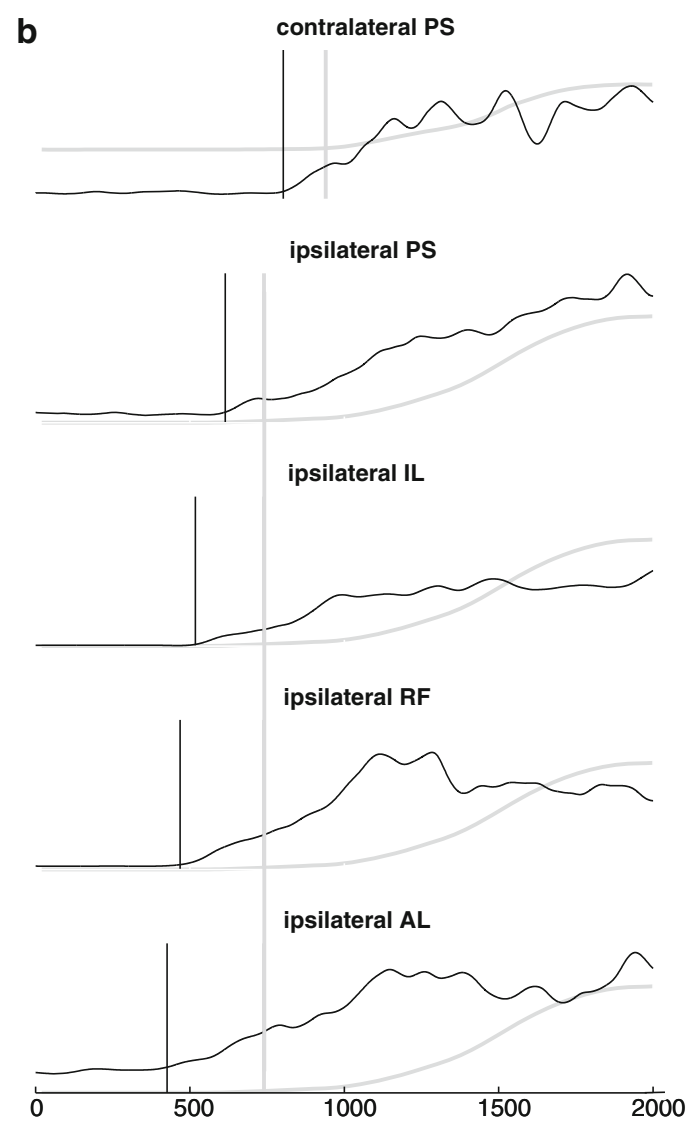

muscle names and side (see Fig. 2b). b Greater temporal resolution of the onset of muscle activity (black vertical lines), and the onset of movement of the lower leg (grey vertical lines). PS psoas, $I L$ iliacus, $R F$ rectus femoris, $A L$ adductor longus

is a hip flexor. In principle, this is consistent with the psoas activity found during ipsilateral ASLR. However, psoas was also active during contralateral ASLR, with similar amplitude and onset time. This pattern did not change when weight was added above the ankle. These results imply that psoas is not used as a hip flexor contralaterally. Rather than invoking different explanations for ipsilateral and contralateral psoas activity, the simplest account would be that psoas is recruited bilaterally for some other function than hip flexion [21].

Juker et al. [13] studied psoas activity in a wide variety of tasks, and the muscle was found to be most active in standing, when raising the ipsilateral knee and flexing the hip, while the upper leg was pushed down with the hands. Unfortunately, however, contralateral activity was not measured. In the present study, the psoas was also active contralaterally.

Andersson et al. [1] studied psoas activity in seven subjects, who performed a large number of tasks. The psoas was found to be active during an ipsilateral dynamic 
Table 2 Regression coefficients $(B)$ and $P$ values from GEEs on the onset time of muscle activity during the ASLR, for seven relevant muscle pairs, with Muscle (the first vs. the second muscle in the pair) and Condition (without vs. with weight added) as factors

\begin{tabular}{|c|c|c|c|c|c|c|c|c|}
\hline \multirow[t]{2}{*}{ Comparison } & \multicolumn{2}{|l|}{ Intercept } & \multicolumn{2}{|l|}{ Muscle } & \multicolumn{2}{|c|}{ Condition } & \multicolumn{2}{|c|}{ Interaction } \\
\hline & $B$ & $P^{\mathrm{a}}$ & $B^{\mathrm{b}}$ & $P^{\mathrm{a}}$ & $B^{\mathrm{c}}$ & $P^{\mathrm{a}}$ & $B^{\mathrm{d}}$ & $P^{\mathrm{a}}$ \\
\hline \multicolumn{9}{|c|}{ Ipsilateral versus contralateral } \\
\hline PS versus PS & -117.94 & 0.00 & 53.63 & 0.15 & -61.74 & 0.00 & & \\
\hline \multicolumn{9}{|c|}{ Ipsilateral versus ipsilateral } \\
\hline PS versus IL & -118.52 & 0.00 & -68.60 & 0.01 & -47.09 & 0.00 & & \\
\hline PS versus RF & -116.99 & 0.00 & -56.51 & 0.01 & -51.19 & 0.00 & & \\
\hline PS versus $A L$ & -106.10 & 0.00 & -74.27 & 0.01 & -67.28 & 0.00 & & \\
\hline IL versus RF & -195.88 & 0.00 & 14.46 & 0.47 & -35.32 & 0.03 & & \\
\hline IL versus $A L$ & -193.79 & 0.00 & 14.68 & 0.62 & -31.59 & 0.04 & -38.23 & 0.02 \\
\hline RF versus $A L$ & -172.07 & 0.00 & -14.93 & 0.37 & -54.04 & 0.00 & & \\
\hline
\end{tabular}

Cf. also Table 1

$P S \mathrm{~m}$. psoas, $I L \mathrm{~m}$. iliacus, $R F \mathrm{~m}$. rectus femoris, $A L \mathrm{~m}$. adductor longus

${ }^{a}$ For the model effects (which may be different from $P$ values for specific parameterizations)

${ }^{\mathrm{b}}$ For the muscle mentioned last in the first column

${ }^{\mathrm{c}}$ For the condition with weight added

${ }^{\mathrm{d}}$ For the muscle mentioned last in the first column, in conditions with weight added

straight leg raise, up and down $60^{\circ}$, but silent contralaterally. This result appears to be in contrast with the present study. Psoas recruitment is task-dependent [2], and maybe the dynamic straight leg raise up to $60^{\circ}$ is a different task from the ASLR in the present study. Perhaps, there are inter-individual differences in the details of motor control, task performance, and/or initial position. Post hoc we inspected psoas activity signals of the 13 subjects with high quality EMG data. In three subjects, there was more ipsilateral than contralateral psoas activity, in four ipsilateral activity was less, and in six activities appeared equal. Still, such inter-individual variability cannot explain the difference with the study of Andersson et al. [1], who reported no contralateral activity, while the present study found contralateral activity in all subjects.

\section{Spinal stabilization}

Because the psoas connects the lumbar spine with the femur, it can affect not only the hip joint, but also the lumbar spine, and, more indirectly, the pelvis. During ASLR, the psoas may be recruited to stabilize the lumbar spine.

Nachemson [17, 18] suggested that psoas contraction stabilizes the spine, thereby adding to compressive forces, which stiffen spinal joints [9]. In the frontal plane, psoas can be used for lateral stabilization [1], and bilateral psoas activation appears to work as guy wires stabilizing the spine as if it were a mast of a ship [21]. In the sagittal plane, psoas might contribute to the regulation of lumbar lordosis [19], and in the transverse plane, the role of psoas in spinal stabilization is dependent upon the exact parameters of the task at hand [3].

If subjects raise a leg from the supine position, gravity will exert a considerable moment, and many muscles will be involved [12]. Thus, ASLR may perturb the lumbar spine. Although the mechanisms to stabilize the lumbar spine are outside the scope of the present study, it appears plausible that bilateral psoas activation during ASLR served to stabilize the lumbar spine, particularly in the frontal plane. Nikolai Bernstein once wrote: "There are no situations in which muscle shortening is the cause of a movement" [5, p. 119], highlighting that one always has to take the whole interplay of forces into account. In ASLR, psoas activity cannot be understood unless, amongst other factors, gravity is accounted for, the activity of other muscles, and the need to stabilize the lumbar spine.

\section{Limitations}

The determination of EMG onset time is sensitive to noise, and in the present study, other technical problems were also encountered, probably related to instability of connections. Nevertheless, all usable psoas signals showed contralateral activity, and the results of statistical analysis clearly suggested that psoas activity was bilateral. It is highly unlikely that technical problems systematically affected our main conclusions.

Fine-wire EMG records activity from a small region of the muscle, in the present study at the level of L3-L4 [1], and it may be desirable to replicate the study for other parts 
of the psoas. More important, the range of hip flexion was limited in ASLR, well below the $45^{\circ}$ where hip flexion was argued to become a major function of psoas [25]. It will be important to replicate the present study with larger amounts of hip flexion.

Initial trunk position was not controlled, and possible frontal plane deviations in the kinematics of the raising leg were not recorded. These factors may have contributed to inter-individual variation.

\section{Conclusions}

In the ASLR, the ipsilateral iliacus, rectus femoris, adductor longus, and psoas are active, as is the contralateral psoas. Contralaterally, the psoas cannot be used as a hip flexor. The psoas is probably active bilaterally to stabilize the lumbar spine in the frontal plane.

Acknowledgments Financial support was obtained from Stryker Howmedica Nederland, Biomet Nederland, and the Dutch Society of Exercise Therapists Cesar and Mensendieck (VvOCM). The authors gratefully acknowledge Erwin van Wegen, Mark Scheper, Ilse van Dorst, Kitty Bos, Annemarie ten Cate, Margot Prins, and Hans van den Berg (Biomet Nederland) for their help and suggestions. This project could not have been performed without the stimulating initiative of the late Paul I.J.M. Wuisman, Professor of Orthopaedic Surgery at the VU University Medical Centre.

Open Access This article is distributed under the terms of the Creative Commons Attribution Noncommercial License which permits any noncommercial use, distribution, and reproduction in any medium, provided the original author(s) and source are credited.

\section{References}

1. Andersson E, Oddsson L, Grundström H, Thorstensson A (1995) The role of the psoas and iliacus muscles for stability and movement of the lumbar spine, pelvis and hip. Scand J Med Sci Sports 5:10-16

2. Andersson EA, Nilsson J, Thorstensson A (1997) Intramuscular EMG from the hip flexor muscles during human locomotion. Acta Physiol Scand 161:361-370

3. Andersson EA, Grundström H, Thorstensson A (2002) Diverging intramuscular activity patterns in back and abdominal muscles during trunk rotation. Spine 27:E152-E160

4. Basmajian JV (1958) Electromyography of iliopsoas. Anat Rec $132: 127-132$

5. Fel'dman A, Meijer OG (eds) (1999) Discovering the right questions in motor control: movements (1929). Motor Control 3:105-134

6. Bogduk N, Pearcy M, Hadfield G (1992) Anatomy and biomechanics of psoas major. Clin Biomech 7:109-119

7. Bogduk N (1997) Clinical anatomy of the lumbar spine and sacrum, 3rd edn. Churchill Livingstone, Edinburgh

8. De Groot M, Pool-Goudzwaard AL, Spoor CW, Snijders CJ (2008) The active straight leg raising test (ASLR) in pregnant women: differences in muscle activity and force between patients and healthy subjects. Man Ther 13:68-74

9. Garnder-Morse MG, Stokes IA (2003) Physiological axial compressive preloads increase motion segment stiffness, linearity and hysteresis in all six degrees of freedom for small displacements about the neutral posture. J Orthop Res 21:547-552

10. Hides JA, Fan T, Stanton WR, Stanton P, McMahon K, Wilson S (2010) Psoas and quadratus lumborum muscle asymmetry among elite Australian football league players. $\mathrm{Br} \mathrm{J}$ Sports Med 44:563-567

11. Hodges PW, Bui BH (1996) A comparison of computer-based methods for the determination of onset of muscle contraction using electromyography. Electroencephalogr Clin Neurophysiol 101:511-519

12. Hu H, Meijer OG, Van Dieën JH, Hodges PW, Bruijn SM, Strijers RL, Nanayakkara PW, Van Royen BJ, Wu WH, Xia C (2010) Muscle activity during the active straight leg raise (ASLR), and the effects of a pelvic belt on the ASLR and on treadmill walking. J Biomech 43:532-539

13. Juker D, McGill S, Kropf P, Thomas S (1998) Quantitative intramuscular myoelectric activity of lumbar portions of psoas and the abdominal wall during a wide variety of tasks. Med Sci Sports Exerc 30:301-310

14. Keagy RD, Brumlik J, Bergan JL (1966) Direct electromyography of the psoas major muscle in man. J Bone Joint Surg Am 48:1377-1382

15. Marrè-Brunenghi G, Camoriano R, Valle M, Boero S (2008) The psoas muscle as cause of low back pain in infantile cerebral palsy. J Orthop Traumatol 9:43-47

16. Mens JMA, Vleeming A, Snijders CJ, Stam HJ, Ginai AZ (1999) The active straight leg raising test and mobility of the pelvic joints. Eur Spine J 8:468-473

17. Nachemson A (1966) Electromyographic studies on the vertebral portion of the psoas muscle; with special reference to its stabilizing function of the lumbar spine. Acta Orthop Scand 37:177-190

18. Nachemson A (1968) The possible importance of the psoas muscle for stabilization of the lumbar spine. Acta Orthop Scand 39:47-57

19. Penning L (2000) Psoas muscle and lumbar spine stability: a concept uniting existing controversies. Critical review and hypothesis. Eur Spine J 9:577-585

20. Potvin JR, Brown SH (2004) Less is more: high pass filtering, to remove up to $99 \%$ of the surface EMG signal power, improves EMG-based biceps brachii muscle force estimates. J Electromyogr Kinesiol 14:389-399

21. Santaguida PL, McGill SM (1995) The psoas major muscle: a three-dimensional geometric study. J Biomech 28:339-345

22. Standring S (ed) (2008) Gray's anatomy: the anatomical basis of clinical practice, 40th edn. Churchill Livingstone, London

23. Staude G, Wolf W (1999) Objective motor response onset detection in surface myoelectric signals. Med Eng Phys 21:449-467

24. Staudenmann D, Potvin JR, Kingma I, Stegeman DF, van Dieën JH (2007) Effects of EMG processing on biomechanical models of muscle joint systems: sensitivity of trunk muscle moments, spinal forces, and stability. J Biomech 40:900-909

25. Yoshio M, Murakami G, Sato T, Sato S, Niryasu S (2002) The function of the psoas major muscle: passive kinetics and morphological studies using donated cadavers. J Orthop Sci 7:199-207

26. Wu WH, Meijer OG, Uegaki K, Mens JM, Van Dieën JH, Wuisman PIJM, Östgaard HC (2004) Pregnancy-related pelvic girdle pain (PPP), I: terminology, clinical presentation, and prevalence. Eur Spine J 13:575-589

27. Zatsiorsky VM (2002) Kinetics of human motion. Human Kinetics, Champaign 